\title{
A novel low-complexity user selection scheme for multiuser MIMO downlink with zero-forcing beamforming
}

\author{
Le Chen, Sheng Jiang, Shuowen Zhang, Pengcheng Zhu ${ }^{\mathrm{a})}$, \\ and Xiaohu You
}

National Mobile Communications Research Laboratory, Southeast University, Nanjing 210096, China.

a)p.zhu@seu.edu.cn

Abstract: This letter proposed a user selection with swap (USS) algorithm based on zero-forcing beamforming (ZFBF). USS starts with an initial user group with the largest channel 2-norms, iteratively swap the "worst" user (has the minimal achieved rate) among the scheduled users with the "best" user (has the maximum channel 2-norm) among the remaining users. Based on the simulation results, we can confirm that the proposed USS algorithm can provide a comparable performance with previous algorithms with greatly reduced complexity of $\mathcal{O}\left(K N^{2}\right)$.

Keywords: multiuser MIMO, user selection with swap, zero-forcing Classification: Wireless Communication Technologies

\section{References}

[1] Z. Tu and R. Blum, "Multiuser diversity for a dirty paper approach," IEEE Commun. Lett., vol. 7, no. 8, pp. 370-372, 2003.

[2] G. Dimic and N. Sidiropoulos, "On downlink beamforming with greedy user selection: performance analysis and a simple new algorithm," IEEE Trans. Signal Process., vol. 53, no. 10, pp. 3857-3868, 2005.

[3] S. Huang, H. Yin, J. Wu, and V. Leung, "User selection for multi-user mimo downlink with zero-forcing beamforming," IEEE Trans. Veh. Technol., vol. 62, no. 7, pp. 3084-3097, 2013.

[4] T. Yoo and A. Goldsmith, "On the optimality of multiantenna broadcast scheduling using zero-forcing beamforming," IEEE J. Sel. Areas Commun., vol. 24, no. 3, pp. 528-541, 2006.

[5] T. Ji, C. Zhou, S. Zhou, and Y. Yao, "Low complex user selection strategies for multi-user mimo downlink scenario," Proc. IEEE WCNC, pp. 1532-1537, 2007.

[6] G. Caire and S. Shamai, "On the achievable throughput of a multiantenna gaussian broadcast channel," IEEE Trans. Inf. Theory, vol. 49, no. 7, pp. 1691-1706, 2003.

[7] J. Wang, D. Love, and M. Zoltowski, "User selection with zero-forcing beamforming achieves the asymptotically optimal sum rate," IEEE Trans. Signal Process., vol. 56, no. 8, pp. 3713-3726, 2008. 


\section{Introduction}

Multi-input multi-output (MIMO) communication techniques have brought completely new perspectives on how to communicate over wireless channels. Compared to the single-user setting, the multiuser settings offer more opportunities to exploit. In addition to the choice of when to transmit, there is now an additional choice of which user(s) to transmit to (in the downlink) and the amount of power to allocate between the users. This additional choice provides a further performance gain not found in the single-user scenario.

For Gaussian broadcast multiuser MIMO channels, it was proven that dirty paper coding (DPC) can achieve the capacity region. DPC presubstracts multiuser interference to precode multiple users simultaneously, however due to its prohibitively high complexity, it is difficult to implement in real systems. Many suboptimal user selection schemes have been proposed in the recent literature. Greedy zero-forcing dirty-paper (gZF-DP) is proposed in [1], it shows via simulation that the sum rate of gZF-DP is a significant fraction of the sum capacity. The shortcoming of DP coding is that it requires vector coding, and depending on the SNR. For this reason, some user selection algorithms without DP coding were investigated. For example, the zero-forcing with selection (ZFS) algorithm proposed in [2], starts with an empty user set and adds one user in each iteration to maximize the sum rate increment. In [3], S. Huang et al. proposed a greedy user selection with swap (GUSS) to improve the sum rate performance with a bit higher complexity than ZFS. Semi-orthogonal user selection (SUS) based on searching for a user set with near-orthogonal channel vectors is proposed in [4]. T. Ji et al. proposed a scheme named correlation-based user selection (CBUS) in [5], which is similar to SUS for the case where each user equipped with multi-antenna.

The user selection with swap (USS) proposed in this letter has excellent sum rate performance and keeps low complexity at the meantime.

\section{System model}

Consider a MIMO downlink channel with a single BS and $K$ user terminals. The BS is equipped with $N(N<K)$ transmit antennas, and each user terminal with a single receive antenna. The maximum number of simultaneous users supported by the BS is $M(M \leq N)$. We use a simple channel model where the channel gain from a transmit antenna to a user is described by a zero-mean circularly symmetric complex Gaussian (ZMCSCG) random variable. Denote the $j$ th scheduled user by $u_{j}$ and the set of scheduled users by $\mathcal{U}$ such that $u_{j} \in \mathcal{U} \subset \mathcal{T}=\{1,2, \ldots, K\}$ and $|\mathcal{U}| \leq M$, the received signal at the scheduled user $u_{j}$ can be expressed as

$$
y_{u_{j}}=\sqrt{P_{u_{j}}} \mathbf{h}_{u_{j}} \mathbf{w}_{u_{j}} s_{u_{j}}+\sum_{k \neq j} \sqrt{P_{u_{k}}} \mathbf{h}_{u_{j}} \mathbf{w}_{u_{k}} s_{u_{j}}+z_{u_{j}}
$$

where $\mathbf{h}_{u_{j}} \in \mathbb{C}^{1 \times N}$ is the channel gain vector to user $u_{j}, \mathbf{w}_{u_{j}} \in \mathbb{C}^{N \times 1}$ is the beamforming vector, $s_{u_{j}} \in \mathbb{C}$ is the unit-norm transmitted data symbol and $P_{u_{j}}$ is the transmit power scaling factor for user $u_{j}$, respectively. $z_{u_{j}} \in \mathbb{C}$ 
is the additive white Gaussian noise (AWGN) at user $u_{j}$ and $y_{u_{j}} \in \mathbb{C}$ is the received signal by user $u_{j}$. We normalize the channel and noise such that the entries of $\mathbf{h}_{u_{j}}$ and $z_{u_{j}}$ have unit variance, and the transmitter has an average power constraint, $\mathcal{E}\left\{\mathrm{xx}^{*}\right\} \leq P$.

\section{Review of zero-forcing beamforming}

ZFBF inverts the channel matrix at the transmitter so that orthogonal channels between transmitter and receivers are created [6], $\mathbf{h}_{u_{j}} \mathbf{w}_{u_{k}}=0$ for $j \neq k$. Define the composite channel matrix $\mathbf{H}_{\mathcal{U}}=\left[\begin{array}{lll}\mathbf{h}_{u_{1}}^{T} & \ldots & \mathbf{h}_{u_{|\mathcal{U}|}}^{T}\end{array}\right]^{T}$, then the ZFBF matrix $\mathbf{W}_{\mathcal{U}}=\mathbf{H}_{\mathcal{U}}^{\dagger}=\mathbf{H}_{\mathcal{U}}^{*}\left(\mathbf{H}_{\mathcal{U}} \mathbf{H}_{\mathcal{U}}^{*}\right)^{-1}$, is the Moore-Penrose pseudoinverse of the channel matrix. Let the columns of $\mathbf{W}_{\mathcal{U}}$ be $\mathbf{w}_{1} \ldots \mathbf{w}_{|\mathcal{U}|}$, i.e., $\mathbf{W}_{\mathcal{U}}=$ $\left[\begin{array}{lll}\mathbf{w}_{1} & \ldots & \mathbf{w}_{\mid \mathcal{U}}\end{array}\right]$. The beamforming vector $\mathbf{w}_{u_{j}}$ can also be obtained through the effective channel vector $(\mathrm{ECV}) \boldsymbol{\nu}_{u_{j}}[3]$

$$
\mathbf{w}_{u_{j}}=\frac{\boldsymbol{\nu}_{u_{j}}^{*}}{\left\|\boldsymbol{\nu}_{u_{j}}\right\|^{2}}, \quad \boldsymbol{\nu}_{u_{j}}=\mathbf{h}_{u_{j}} \mathbf{P}_{u_{j}}^{\perp}
$$

where $\mathbf{P}_{u_{j}}^{\perp}=\mathbf{I}_{N}-\mathbf{H}_{\mathcal{U} \backslash\left\{u_{j}\right\}}^{*}\left(\mathbf{H}_{\mathcal{U} \backslash\left\{u_{j}\right\}} \mathbf{H}_{\mathcal{U} \backslash\left\{u_{j}\right\}}^{*}\right)^{-1} \mathbf{H}_{\mathcal{U} \backslash\left\{u_{j}\right\}}$ is the orthogonal projector matrix on the subspace $V_{u_{j}}=\operatorname{span}\left\{\mathbf{h}_{u_{i}} \mid u_{i} \in \mathcal{U}, i \neq j\right\}, \mathbf{H}_{\mathcal{U} \backslash\left\{u_{j}\right\}}$ is the row-reduced channel matrix of all the selected users except user $u_{j} . \mathcal{U} \backslash\left\{u_{j}\right\}$ denotes the set difference that deletes the element $u_{j}$ from the set $\mathcal{U}$.

The achievable sum rate of user subset $\mathcal{U}$ is given by

$$
R_{Z F B F}(\mathcal{U})=\max _{\sum_{u_{j} \in \mathcal{U}} \gamma_{u_{j}^{1}}^{-1} P_{u_{j}} \leq P} \sum_{u_{j} \in \mathcal{U}} \log _{2}\left(1+P_{u_{j}}\right),
$$

where $\gamma_{u_{j}}=\frac{1}{\left\|\mathbf{w}_{u_{j}}\right\|^{2}}=\left\|\boldsymbol{\nu}_{u_{j}}\right\|^{2}$ is the effective channel gain and $\gamma_{u_{j}}^{-1} P_{u_{j}}$ is the transmit power allocation to user $u_{j}$, thus $P_{u_{j}}$ is the received SNR of user $u_{j}$. The optimal $P_{u_{j}}$ can be easily obtained by water-filling $P_{u_{j}}=\left(\mu \gamma_{u_{j}}-1\right)_{+}$, note that $(x)_{+}$refers to $\max (x, 0)$ and $\mu$ is the water level satisfying

$$
\sum_{u_{j} \in \mathcal{U}}\left(\mu-\gamma_{u_{j}}^{-1}\right)_{+}=P
$$

The highest achievable sum rate of ZFBF can be obtained by exhaustive search (called ZFBF optimal in this letter): For each value of $|\mathcal{U}|$, find all possible user subsets $\mathcal{U}$ and select the one yields maximum $R_{Z F B F}(\mathcal{U})$.

\section{Proposed algorithm: USS}

\section{1 $\gamma$ updating scheme for swapping}

According to $(3) \sim(4)$, the effective channel gain $\gamma$ is the key parameter in evaluating the sum rate of the selected user set $\mathcal{U}$. The complexity reduction method in ZFS updates $\gamma$ via iteratively updating $\mathbf{H}_{\mathcal{U}}^{\dagger}$ and is only applicable when a new user is added to $\mathcal{U}$. In [3], the authors presented an efficient $\gamma$ updating strategy which is suitable for swapping via iteratively updating ECV $\boldsymbol{\nu}$ defined in (2). This updating strategy will be adopted in our USS algorithm to further reduce the complexity. 


\subsection{Construction of USS algorithm}

The algorithm works as follows: 1). Select $M$ users with the largest channel gains as the initial user subset $\mathcal{U}(|\mathcal{U}|=M)$, and denote the remaining users as $\overline{\mathcal{U}}(\overline{\mathcal{U}}=\mathcal{T} \backslash \mathcal{U},|\overline{\mathcal{U}}|=K-M)$, then calculate the ZFBF matrix and waterfilling power allocation. Mark the user with the minimum achieved rate $^{1}$ as $u_{\text {min }}$, and evaluate the achieved sum rate $\left.R_{0} .2\right)$. Iteratively swap the $u_{\text {min }}$ in $\mathcal{U}$ with the user $\bar{u}_{\max }$ (the user with maximum channel gain in $\overline{\mathcal{U}}$ ), if the achieved sum rate is larger than $R_{0}$, set it to $R_{0}$, remark $u_{\text {min }}$; else, undo the swap operation. No matter the if condition is satisfied or not, the user $\bar{u}_{\max }$ will be removed from $\overline{\mathcal{U}}$. 3). After $K-M$ times swap operations, all users in $\overline{\mathcal{U}}$ are removed while $\mathcal{U}$ always holds $M$ users. Considering that when the channel is poor conditioned, less than $M$ users scheduled simultaneously may achieve a larger sum rate, we try: delete the user $u_{\text {min }}$, if the achieved sum rate of the $|\mathcal{U}|-1$ users is larger than $R_{0}$, set it to $R_{0}$, continue the deletion till $|\mathcal{U}|=1$, otherwise, finish the algorithm. The detailed USS scheduling process is described below.

\section{1) Initialization:}

- Calculate 2-norms $\left\|\mathbf{h}_{i}\right\|^{2}$, sort in descending order, and denote the ordered user index $1,2, \ldots, K$, then

$$
\mathcal{U} \leftarrow\left\{u_{1}, \ldots, u_{M}\right\} \quad \overline{\mathcal{U}} \leftarrow\left\{u_{M+1}, \ldots, u_{K}\right\}
$$

- Initialize ZFBF vector $\mathbf{w}_{u_{j}}, \boldsymbol{\nu}_{u_{j}}$ and $\gamma_{u_{j}}$

$$
\begin{aligned}
& {\left[\mathbf{w}_{u_{1}} \ldots \mathbf{w}_{u_{M}}\right]=\mathbf{H}_{\mathcal{U}}^{\dagger}=\mathbf{H}_{\mathcal{U}}^{*}\left(\mathbf{H}_{\mathcal{U}} \mathbf{H}_{\mathcal{U}}^{*}\right)^{-1}} \\
& \gamma_{u_{j}}=\frac{1}{\left\|\mathbf{w}_{u_{j}}\right\|^{2}}, \quad \boldsymbol{\nu}_{u_{j}}=\gamma_{u_{j}} \mathbf{w}_{u_{j}}^{*}
\end{aligned}
$$

- Water-filling, denote the achieved rate $R_{0}^{u_{j}}$ for $u_{j}, R_{0}=\sum_{u_{j} \in \mathcal{U}} R_{0}^{u_{j}}$ for sum rate.

- Find the user $u_{\text {min }}$, such that $u_{\text {min }}=\arg \min _{u_{j} \in U} R_{0}^{u_{j}}$.

- Set $k_{1}=k_{2}=1$.

2) While $k_{1} \leq K-M$ :

- Swap user $u_{\min }$ in $\mathcal{U}$ with the first user in $\overline{\mathcal{U}}$, denote as $\bar{u}_{\text {max }}$. Update:

$$
\mathcal{U} \leftarrow \mathcal{U} \backslash\left\{u_{\min }\right\}, \mathcal{U} \leftarrow \mathcal{U} \cup\left\{\bar{u}_{\max }\right\}, \overline{\mathcal{U}} \leftarrow \overline{\mathcal{U}} \backslash\left\{\bar{u}_{\max }\right\}
$$

- Update $\mathbf{w}_{u_{j}}, \boldsymbol{\nu}_{u_{j}}$ and $\gamma_{u_{j}}$ utilizing the updating scheme presented in [3], denote the achieved rate $R_{k_{1}}^{u_{j}}$ for $u_{j}, R_{k_{1}}=\sum_{u_{j} \in \mathcal{U}} R_{k_{1}}^{u_{j}}$ for sum rate.

- If $R_{k_{1}}>R_{0}$

$$
\text { Update: } u_{\text {min }} \leftarrow \arg \min _{u_{j} \in \mathcal{U}} R_{k_{1}}^{u_{j}}, R_{0} \leftarrow R_{k_{1}} \text {. }
$$

- Else, Undo the swap operation.

- Increase $k_{1}$ by 1 .

3) While $k_{2} \leq M-1$ :

- Delete user $u_{\min }$, update: $\mathcal{U} \leftarrow \mathcal{U} \backslash\left\{u_{\min }\right\}$.

- Update $\mathbf{w}_{u_{j}}, \boldsymbol{\nu}_{u_{j}}$ and $\gamma_{u_{j}}$ utilizing the updating scheme presented in [3], denote the achieved rate $R_{k_{2}}^{u_{j}}$ for $u_{j}, R_{k_{2}}=\sum_{u_{j} \in \mathcal{U}} R_{k_{2}}^{u_{j}}$ for sum rate.

\footnotetext{
${ }^{1}$ Mark the user with minimum $\gamma$ as $u_{\min }$ will be equivalent.
} 
Update: $u_{\min } \leftarrow \arg \min _{u_{j} \in \mathcal{U}} R_{k_{2}}^{u_{j}}, R_{0} \leftarrow R_{k_{2}}$.

Increase $k_{2}$ by 1 .

- Else, Break.

4) Return: $R_{U S S}=R_{0}$.

\subsection{Complexity analysis}

In USS algorithm, the initialization in step 1) requires evaluating $K$ 2-norms $\left\|\mathbf{h}_{i}\right\|$ of $1 \times N$ vectors and $M$ 2-norms $\left\|\mathbf{w}_{u_{j}}\right\|$ of $N \times 1$ vectors, which includes $N(K+M)$ complex multiplications. The complexity of USS lies mainly in the Moore-Penrose pseudo-inverse of $\mathbf{H}_{\mathcal{U}}$ in (5), which involves a complexity of $\mathcal{O}\left(N M^{2}\right)$. The 'swap' operation in step 2) requires evaluating $K-M$ sum rates $R_{k_{1}}$. According to equations (45)-(48) of [3], each $R_{k_{1}}$ involves $N M+4 N$ complex multiplications. Repeating this over $K-M$ users in $\overline{\mathcal{U}}$, we obtain the complexity of step 2) as $(K-M)(N M+4 N)$ complex multiplications. The 'delete' operation in step 3) requires evaluating $M-1$ sum rates $R_{k_{2}}$. According to equations (40)-(41) of [3], each $R_{k_{2}}$ needs $M-k_{2} \gamma_{u_{j}}$ updates in total, which requires $M-k_{2}$ vector-vector multiplications, i.e., involves $N\left(M-k_{2}\right)$ complex multiplications. Repeating this over $M-1$ users in $\mathcal{U}$, step 3) requires $\sum_{k_{2}=1}^{M-1} N\left(M-k_{2}\right)$ complex multiplications. The overall complexity of USS $C O M_{1}$ can be written as

$$
\begin{aligned}
C O M_{1} \approx & \underbrace{\mathcal{O}\left(N M^{2}\right)+N(K+M)}_{1^{\text {st }}}+\underbrace{(K-M)(M+4) N}_{2^{\text {nd }}}+\underbrace{\sum_{k_{2}=1}^{M-1} N\left(M-k_{2}\right)}_{3^{\text {rd }}} \\
& <\mathcal{O}\left(K N^{2}\right) \quad(\text { Note }: M \leq N, N<K)
\end{aligned}
$$

GUSS improved the performance with 1.51 to 2.29 times the complexity of ZFS [3], and the complexity of ZFS algorithm proposed in [2] is approximately $\mathcal{O}\left(K N^{3}\right)$. As for the SUS algorithm proposed in [4], its complexity depends heavily on parameter $\alpha$, a smaller $\alpha$ reduces the complexity by eliminating more users not semiorthogonal to the selected user from further consideration in subsequent iterations. For a fixed $\alpha$, the complexity of SUS algorithm is roughly $\mathcal{O}\left(K N^{3}\right)$ [7]. Therefore, the proposed USS algorithm has much lower complexity than all the 3 previous user selection algorithms.

Our simulations in section 5 adopt the optimal $\alpha$ for SUS (called SUS Optimal in this letter) in the way:

- Set $\mathcal{A}=\{1:-0.05: 0.05\}$

- For $i=1, \ldots,|\mathcal{A}|$ :

Run SUS algorithm with $\alpha_{i} \in \mathcal{A}$, Denote the achieved sum rate $R_{S U S}^{i}$.

- Return: $R_{S U S}=\max _{i} R_{S U S}^{i}$.

The complexity of SUS Optimal will be $|\mathcal{A}|$ times of SUS with a fixed $\alpha$.

\section{Simulation results}

In this section, numerical results obtained by Monte Carlo are presented. Let $10 \log _{10} P$ denote the SNR, where the noise variance of each user is assumed 
equal to 1 . The sum rates are averaged over 1000 independent channels. We compare the sum-rate performance of various user selection schemes in Fig. 1. It shows that the proposed USS scheme has almost the same performance with GUSS, ZFS and SUS Optimal, and the sum rate achieved by the 4 schemes are very close to the exhaustive search scheme (ZFBF Optimal) especially when the number of total users is much larger than the number of maximum simultaneous users $(K \gg M)$ or the number of transmit antennas $N>M$.

It is shown in Fig. 2 that the sum rate performance of SUS algorithm is greatly influenced by parameter $\alpha$. If $\alpha$ is too small, the multiuser diversity gain decreases, while if $\alpha$ is too large, effective channel gains are reduced due the loss associated with zero-forcing channel inversion [4]. Even with the optimal $\alpha$ at the cost of high complexity, SUS algorithm didn't provide any performance gain compared with our proposed USS algorithm. With a large $K$ and $N$, USS algorithm has a more attractive complexity advantage while keeping its excellent sum rate performance as shown in Fig. 3.

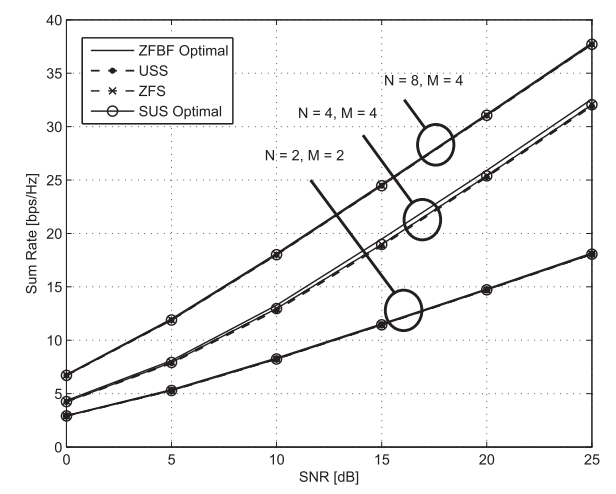

(a) USS vs. ZFBF Optimal, ZFS and SUS Optimal

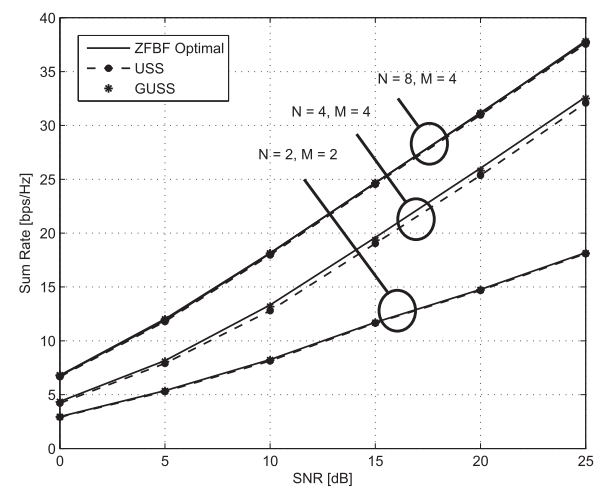

(b) USS vs. ZFBF Optimal and GUSS

Fig. 1. Sum rate performance comparison with $K=16$.

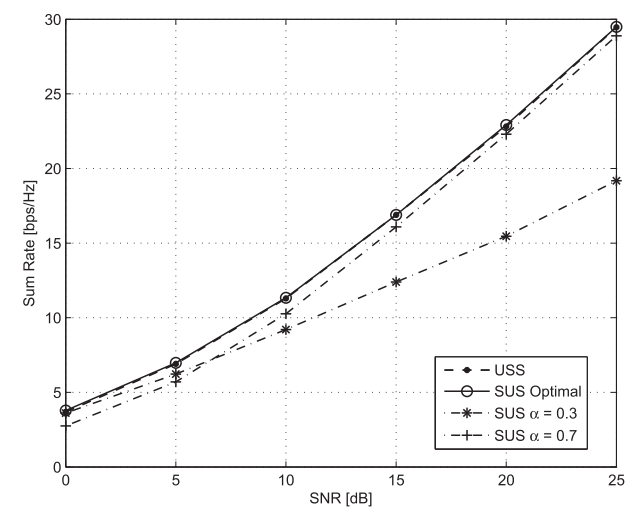

Fig. 2. Sum rate performance comparison with $K=8$, $N=4$ and $M=4$. 


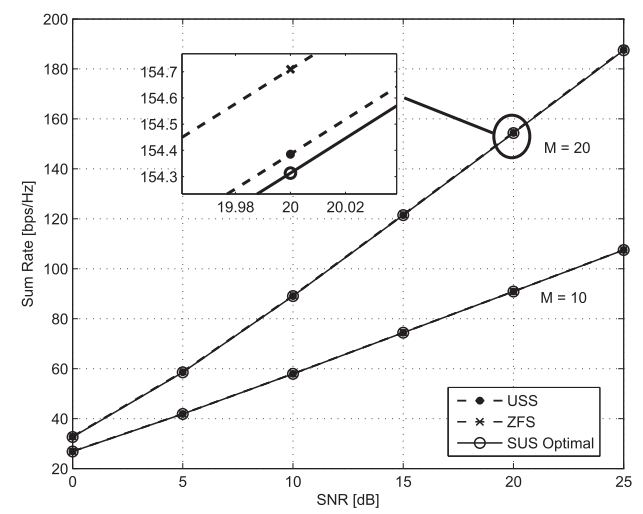

Fig. 3. Sum rate performance comparison with $K=100$, $N=50$ and $M=10,20$.

\section{Conclusions}

We have proposed a novel user selection algorithm, USS, which achieves a significant fraction of the ZFBF Optimal sum rate. We have shown that the selection procedures of USS have the complexity order $\mathcal{O}\left(K N^{2}\right)$, which is much lower than the previous user selection algorithms especially when $K$ and $N$ goes to large. We have compared the sum rate performance of the proposed USS algorithm with GUSS, ZFS and SUS (Optimal $\alpha$ is used), it performs well in all the simulations, even slightly better than SUS Optimal.

\section{Acknowledgement}

This work is supported by the National Key Special Program of China under grant 2012ZX03001036-004. 